\title{
Improvement of L-Valine Production by Atmospheric and Room Temperature Plasma Mutagenesis and High-Throughput Screening in Corynebacterium glutamicum
}

Guoqiang Han ${ }^{1,3 \#}$, Ning $\mathrm{Xu}^{2 \#}$, Xieping Sun ${ }^{1}$, Jinzhao Chen ${ }^{1}$, Chun Chen ${ }^{1}$, Qing Wang ${ }^{1,3^{*}}$

${ }^{1}$ Life Science and Technology Institute, Yangtze Normal University, P.R. China;

2 Tianjin Institute of Industrial Biotechnology, Chinese Academy of Sciences, Tianjin, P.R. China;

${ }^{3}$ School of advanced agriculture and bioengineering, Yangtze Normal University, P.R. China.

${ }^{*}$ Corresponding author:

Qing Wang

Life Science and Technology Institute

Yangtze Normal University

16 Juxian Avenue Fuling

Chongqing 408100

People's Republic of China

Tel: +86-23-72792193

E mail: 767783845@qq.com

\# These authors contributed equally to this work. 
Supplementary tables and figures

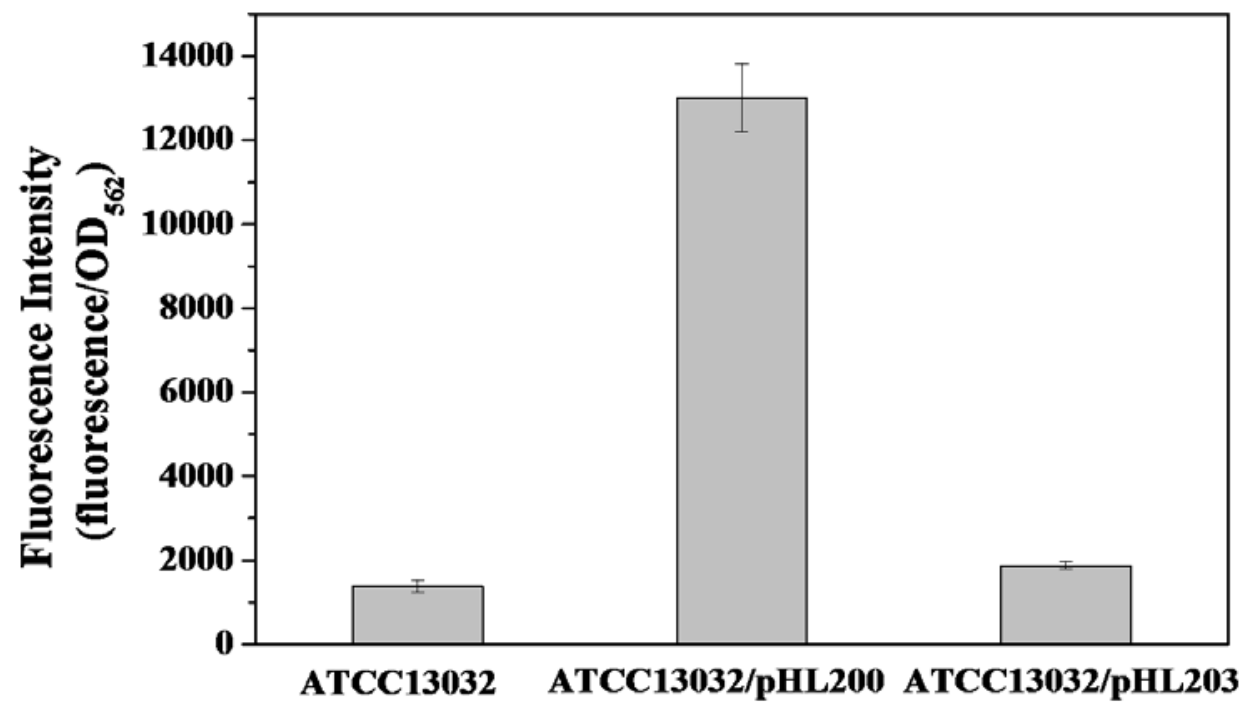

Fig. S1 Fluorescence intensity of pHL203 and pHL200. The data represent the means \pm SEM $(\mathbf{n}=3)$.

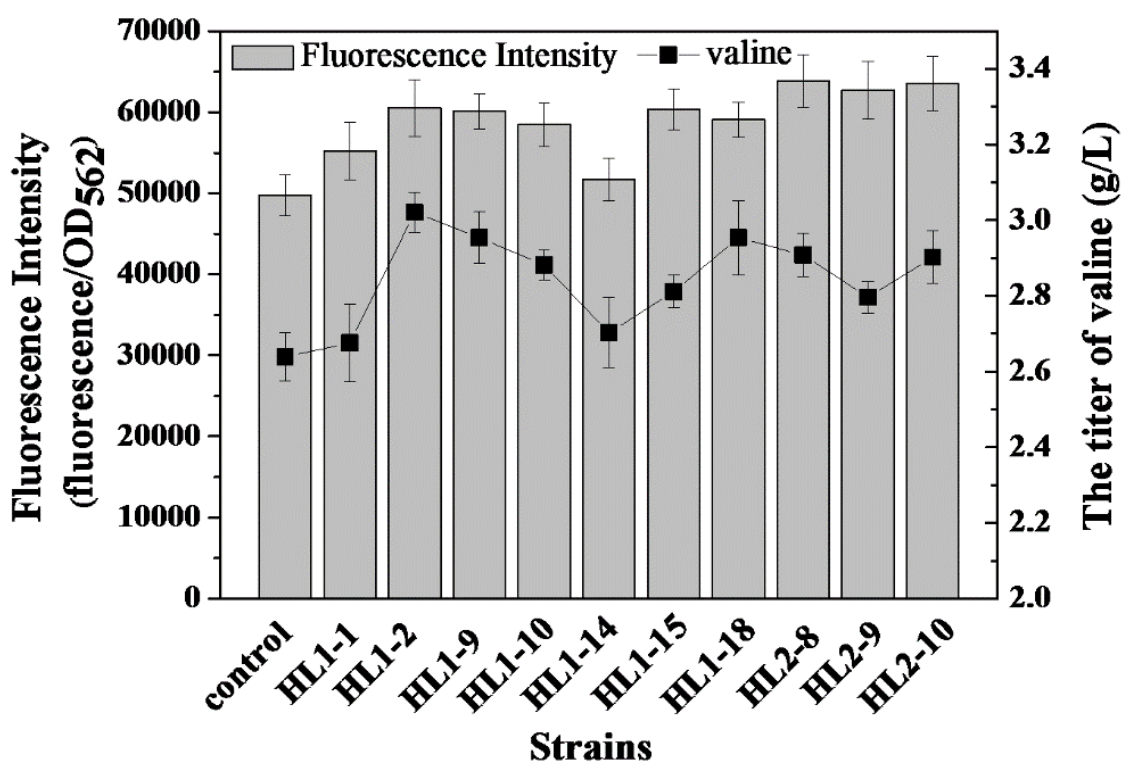

Fig. S2 Normalized fluorescence intensity and Fermentation analysis of $C$. glutamicum mutants. The control is strain HL104/pHL203. The data represent the means \pm SEM $(n=3)$. 
Table S1 SV test, SNP test and Small InDel test results

\begin{tabular}{|c|c|c|c|c|c|c|c|c|c|}
\hline & \multicolumn{4}{|c|}{ SV } & \multicolumn{3}{|c|}{ SNP } & \multicolumn{2}{|c|}{ Small InDel } \\
\hline & INS & DEL & INV & ITX & Ts & $\mathrm{Tv}$ & $\mathrm{Ts} / \mathrm{Tv}$ & Insertion & Deletion \\
\hline HL1-6 & 63 & 4 & 11 & 3 & 14 & 6 & 2.33 & 1 & 1 \\
\hline HL1-19 & 57 & 5 & 9 & 3 & 13 & 7 & 1.86 & 2 & 1 \\
\hline HL1-20 & 52 & 5 & 13 & 7 & 22 & 8 & 2.75 & 2 & 0 \\
\hline HL2-5 & 38 & 4 & 8 & 6 & 16 & 7 & 2.29 & 3 & 1 \\
\hline HL2-7 & 42 & 6 & 9 & 5 & 14 & 9 & 1.56 & 2 & 1 \\
\hline HL2-11 & 45 & 2 & 3 & 4 & 17 & 8 & 2.13 & 2 & 0 \\
\hline HL2-12 & 35 & 3 & 2 & 3 & 20 & 7 & 2.86 & 2 & 0 \\
\hline HL2-17 & 46 & 6 & 4 & 8 & 13 & 7 & 1.86 & 3 & 1 \\
\hline
\end{tabular}

SV, Structural Variation; INS, insertion; DEL, deletion; INV, inversion; ITX, intra-chromosomal translocation; SNP, single nucleotide polymorphism; Ts, Transitions; Tv, Transversions. 